\title{
Tackling Foundations of Systemic Complexity on Reality and Decision Systems
}

\author{
Hermínio Duarteramos \\ Emeritus Professor \\ New University of Lisbon \\ Lisbon \\ hduarteramos@gmail.com
}

\begin{abstract}
What is reality? That's an old question, a big question raised by so many thinkers, since very ancient times. Many responses has been given, so many answers are purposed in these days. Here we advance on quantum territories and we also approach the human decision theory to interpret the complex meaning of reality under new systemic complexity viewpoints.
\end{abstract}

Keywords: reality, systemic complexity, decision system, QBism, Bayesian probability.

Traditionally, thoughts on reality were put forward among philosophers and scientists, around force lines of idealism and materialism dipoles, as Plato (427-347 BC) and Lucretius (99-55 BC) traced on matter, or over generated currents between theism and deism bipoles, as Wilhelm Leibniz (1646-1716) and Albert Einstein (1879-1955) did conduct about the universe [1].

Today we find some other ways of thinking, based on new scientific interpretations, using abstract fields of mathematics, as John von Neumann (1903-1957) supposing a consciousness creation for object attributes, and quantum physics, as Niels Bohr (1885-1962) negating deep reality down phenomenal facts [2].

Nowadays, what can we say about the meaning of reality?

First of all, it is a general topic, which appears inevitably at the front panel of globalization's science, thinking on worldwide vertiginous trends of scientific research without national frontiers.

The reality meaning is a complex problem to understand systems we meet on nature, focusing our consciousness on things, because we aren't aware of essentials on material structures and human-nature interactions.

Nevertheless, today we know much better what reality can be than some centuries ago, and even decades behind or from late years.

Here I join three basic ideas, integrating them with the general system theory to interpret what reality is, namely the wavefunction for the quantum formulation of the ultimate matter, the complexity of an observed system and the subjective probability on decision making.

In such way, I briefly tackle how to set up general foundations of the so called systemic complexity, hopping to contribute with a new and powerful vision on system sciences.

It is inevitable to approach physical and mathematical concepts, at least evocating some descriptive principles, because physics and mathematics are sciences of the reality modelling, that humans use to understand the world around.

What is reality?

Outside my mind there are things on the objective (or concrete) world, which are only matter and signals, and inside the mind it runs a subjective (or abstract) world with forms of ideas and signs ${ }^{1}$.

The human detects signals that are radiated from matter and he sees or hears the impressive reality, and then he judges on what he is seeing or hearing by decision making on some important cues, which are detected signals contributing to the awareness of reality.

On one hand, the physics (the natural philosophy in past times) measures and relates features of the objective reality, since electromagnetic waves as signals of light until quantum waves as signals of the ultimate matter, i.e., the physics objectivity interprets the external reality by its fundamental properties, and so the objectivity in physics doesn't describe

\footnotetext{
${ }^{1}$ We don't discuss here the mind's cognition nature of brain's imagerial and soul's imaginal information processes.
} 
the world "as it really is" [3], but it is an epistemic knowledge devoted to measurable features of the world, on which an open worldwide community of independent observers do agree.

On the other hand, cognition sciences (encompassing the brain, the mind and the soul) study features of the subjective reality, since sensing signals and their perception until the ultimate decision on inner appearances, i.e., the cognitive subjectivity also represents interpretations on the outer reality.

When Plato's prisoners emerged from the cave, they were only able to make out shadows and reflexions as reality, not just because the sunlight burned their eyes but also due to the subjective probability of decision making in the brain's mind.

Within this frame, I wish to participate freely on the open discussion of some fundamental thoughts on complexity, to better understand systems both of objective and subjective realities, trying to discover an all-embracing model for a single scientific paradigm of reality systems.

Each one of us makes decisions taking systems from the natural matter and observing their behaviour, and then we interpret what it is going on actually in our mind as a real representation.

How can we do that?

Basically, a system is one concrete structure of several interconnected parts within a limited space and interacting in concrete modes (doesn't a matter what they are at the moment), emerging to us inherent black box outputs ${ }^{2}$, as reactions to eventual stimulating inputs or owing to its own autonomous existence, besides the necessary vital energy feeding things to exist with an own intentionality.

We call systemics the science on the function integration of whatever composite system in order to process its behaviour by a functional model.

In open systems the outputs emerge from the internal operation to the outward environment, and otherwise inward outputs define closed systems.

Generally speaking, the functional organization of a given system is characterized by the following four operational systemic essentials: the acrony or composition, the axony or interactivity, the aquadry or boundary, the adaptacy or optimum workability.

However, the most notable feature is that the real system always denotes an existential intentionality that must be truly expressed by their outputs into the environment, which may be detected by instruments or human observers.

These external information signals establish a quintessential systemic principle, the telonomy or aim outputs representing the intrinsic intentionality of the system in action [4].

That is to say, a system accomplishes its existential purpose if its bulk operation obeys to those five systemic principles, and we need to know them clearly in order to acknowledge its real behaviour.

In complex systems (distinct from complicated ones) we don't know quite well one or more systemic essentials, otherwise the correct knowledge of all these characteristics defines a simplex system (distinct from simple ones).

In the case of a simplex system all systemic essentials are well known, and so we have a definite acrony and complete axony and certain aquadry and determinate adaptacy and an accurate telonomy.

The four operational systemic essentials of a simplex system may be expressed mathematically onto a transfer function ${ }^{3}$ to calculate its right behaviour, denoting the systemic telonomy at each time, but complex systems incorporate additional barriers for obtaining reasonable outcomes, which are variable with the correctness of systemic essentials.

The degree of complexity for the system's observer is related to the imperfect knowledge he has on systemic essentials, for instance, the complexity of a simplex system is zero degree, and a complex system of $2^{\text {nd }}$ degree has two systemic essentials that we don't known entirely, either indefinite acrony or incomplete axony or uncertain aquadry or indeterminate adaptacy or inaccurate telonomy.

The concept of systemic complexity ${ }^{4}$ was introduced to approach complexities encountered in material or concrete (objective) systems, and the same functional methodology was extended to mind or abstract (subjective) systems, both trying to reduce complexity into simplexity.

Here they are some foundations of the systemic complexity of general and singular systems to interpret the observed natural world, fusing accessible conscious information on imagerial and imaginal mind processes.

${ }^{2}$ The system science uses efficiently the concept of "black box" and its "outputs", being equivalent to the whole functional behaviour and ignoring the real system structure.

${ }^{3}$ The transfer function of a system requires initial conditions to be defined in the complex frequency domain $s=\sigma+\mathrm{i} \omega$, where $\sigma$ is a real frequency and $\mathrm{i} \omega$ is the imaginary angular frequency.

${ }^{4}$ The adjective systemic clearly qualifies the complexity we are talking about, distinguishing it from other complexity types as we find in mathematics (Kolmogorov complexity) or in computer sciences (algorithm complexity) and also in physics (pattern complexity) [5]. 
We use it to acknowledge both objective and subjective reality, for the near contact world till the ultimate mater in the void real space.

The world scientific community in the twentieth century did interpret the reality using thoughts derived from the quantum theory, since the famous "Copenhagen interpretation" by the physicist Niels Bohr supposing that reality is created by observation, and the wholeness of David Bohm (1917-1992), until parallel universes purposed by Hugh Everett (1930-1982), and also the world consisting of potentials and actualities under the uncertainty principle of Werner Heisenberg (1901-1976) considering somewhat as probability waves in the middle between possibility and reality, after Louis de Broglie (1892-1987) having translated the reality into a "statistical interpretation" [2].

What is the quantum interpretation of the ultimate reality?

It's known that the wavefunction $\Psi(\boldsymbol{r}, t)$ was formulated by the physicist Erwin Schrödinger (1887-1961) describing the behaviour of elementary particles through an equation ${ }^{5}$, which is interpreted as representing probability values of particle states on its existential trajectory (traced by the space-vector $\boldsymbol{r}$ at each time $t$ ).

This mode of thinking do gives us a way to predict the electron behaviour on the move, calculating its own evolution in space, and pointed out a potential way to interpret the real face of things.

The wavefunction corresponds to the frequency probability ${ }^{6}$ of the state occurrence, which is an objective probability, for instance, it's value is $50 \%$ in case of an electron ${ }^{7}$, because this particle assumes at each time just one natural state between two possible (up/down) spin states.

The logic consequence is that the quantum reality itself has a probabilistic character, while our common world reality seems to exclude a statistical temper.

From that quantum narrative, the so called "Schrödinger's cat paradox" shows an intriguing quantum ambiguity of the cosmic nature, having been very much discussed among thinkers on the quantum world [6].

It can be analysed considering a closed system formed by a cat inside a sealed room, where there is a glass flash full of poison, besides a radioactive source emitting particles into the inner space and also a particle decay detector which will trigger automatically the fall of a heavy hammer on the flash immediately after detecting a preset particle decay threshold, shattering it and pouring the poison out.

Thus, the cat will be killed, but in the meantime the cat was simultaneously alive and dead, according to the wavefunction of the particle represented by state probabilities.

However our direct observation certificates correctly if the cat is alive or not, since it cannot be in both states at the same time.

The paradox lies in the fact that the animal is alive and dead inasmuch we don't observe what is going on inside the hermetic room (the cat doesn't look at the emitted particles for sure), and when an observer looks at the particles he can say rigorously the cat is either alive or dead.

This happens after the superposition of quantum states ends by observation, producing the collapse of the wavefunction, and then it doesn't represent any more an objective probability, being the observed reality inevitably either one or another possible concrete real state.

That was the reason why Albert Einstein felt serious doubts on quantum theory implications, and he did write to his friend Max Bohr (1882-1804) "I cannot believe that God plays dice with the universe" [7].

During decades many scientists endeavoured to explain such logic contradiction of the strange quantum paradox.

Nevertheless, they struggled without success.

Let us now analyse the Schrödinger's cat system under the systemic complexity point of view.

The acrony is definite because we are aware of all components inside the closed room; the axony is complete once interactions will follow exactly some serial causations of the automation mechanism breaking the glass with poison; the aquadry is certain due to rigid boundaries build by room walls; the adaptacy is determined supposing the structure will work perfectly as it was planned.

\footnotetext{
${ }^{5}$ The time dependent Schrödinger equation for a single non-relativistic particle moving in an electric field is
}

where $h$ is the constant's Planck, $m$ is the particle's mass, $V$ is its potential energy, $\nabla$ is the Laplacian, and $\Psi(\boldsymbol{r}, t)$ is the positionspace wavefunction.

${ }^{6}$ Frequency (or objective) probability $p_{\mathrm{o}}$ is defined by the ratio of the number of favourable cases $\left(n_{\mathrm{fav}}\right)$ and the number of possible cases $\left(n_{\text {pos }}\right)$, or mathematically $p_{\mathrm{o}}=n_{\text {fav }} / n_{\text {pos }}$.

${ }^{7}$ The electron is an elementary particle of fixed mass and electric charge (corpuscular reality, discovered in 1897) and also found to be a wave (waving reality, discovered in 1927). 
So it seems to be a simplex system, but we can't attribute a single state to the cat, the quantum ambiguity remains and we continue ignoring the true reality of the system before its observation.

Why?

Because the system is closed, and the systemic theory can be effectively applied only to open systems, measuring their outputs or system telonomy, i.e., the observer belongs to the global system of reality acknowledgement, and he cannot be ignored.

Notice that the so called observer can be a human or an instrument, and for this the observation may correspond to a measurement whose result is a real measure.

In the closed system we can say that the non-observed (or not measured) reality is represented by the state objective probability ascribed to the wavefunction, according to the quantum theory, and the observed reality on the consequent open system is described by a physical state convincing the observer about the reality character.

Before observation, in spite of the observer awareness on the inner operating systemic essentials, he didn't get any external system telonomy and the reality was complex to him.

When we observe the reality of the open system we verify the cat is alive or dead without any doubt, and before observation the reality of the closed system was ambiguous (due to the quantum wavefunction), being the cat alive and dead, although we were conscious that just one of two possibilities could arrive, saying that the cat is either alive or dead.

That's the real problem, a restless and/or question to the reality in those closed/open systems, revealing a fundamental difference between the quantum world, which is an ocean of waves, and the macroscopic world on the human scale, where we live.

At the existential limit of the nature, the quantum theory makes out the reality with all possible objective probability states, and at the human existential nature, the reality to observers means something concrete far from objective probability of real states.

Each part of the reality on our Newtonian world exists each time apparently with only one form, because multiple own states may not superpose at the same time in any physical system.

What is such potential concreteness of our observation?

Maybe it is the old concept potentia of the Aristotle (384-322 BC) philosophy, maybe it isn't.

Recently that contradiction of the quantum theory has been overshoot by the so called QBism ${ }^{8}$, applying the subjective probability of Bayes to the wavefunction.

In the eighteenth century, the English Presbyterian and statistician Thomas Bayes (1701-1716) had a different idea on the probability notion, and in 1812 the French mathematician and astronomer Pierre-Simon, marquis de Laplace (1749-1827), disseminated it among other scientists of the natural philosophy, gaining credibility last century in several statistical applications, as in economics and also engineering decision and in cognition sciences.

In fact, the Bayesian probability ${ }^{9}$ measures the human conviction on estimated cues or contents of signals that we detect from the reality to make decisions.

For instance, it happens when a physician regards his patient and evaluates the symptoms to diagnose a disease, and he would be self believed on the most credible hypothesis, guessing it by means of subjective probabilities, even he uses a practical method giving weights to the collected data.

Such subjective probability quantifies the human conviction level on the observed reality, being entirely different from the objective probability based on illness occurrences, which results from the disease frequency and it is not related to the human convincement about the reality itself.

The modern decision theory has applied successfully the Bayesian probability, conveying an important tool for engineers, economists, sociologists and other users, and even to solve trivial situations we have in our everyday life ourselves.

${ }^{8}$ The term QBism is derived from the Quantum Bayesian view, using the Bayesian probability of elementary particle states in the wavefunction.

${ }^{9}$ Bayesian (or subjective) probability is defined by the Bayes' rule

where I denotes a condition probability; $H$ stands for competing hypothesis (competitors) from which one chooses the most probable; the evidence $E$ (cue) corresponds to new data that were not used in computing the prior probability; the prior probability $p(H)$ is the probability of $H$ before $E$ is observed; the model evidence $p(E)$ or the marginal likelihood (diagnosticy weight) is the same for all possible hypothesis being considered; the likelihood function $p(E \mid H)$ is the probability of observing $E$ given $H$ (impact weight); the posterior probability $p(H \mid E)$ is the probability of $H$ given $E$, i.e., after $E$ is observed (credibility weight); the impact factor $p(E \mid H) / p(E)$ represents the impact of $E$ on the probability of $H$. 
Undoubtedly, we are observers of the reality and making decisions always with subjective probabilities on all things we experience around us.

Now we pay attention to quantum reality supposing that wavefunction superposes subjective (or Bayes) probability states instead objective (or frequency) probability states.

Reasoning like that we don't see any paradox.

Indeed, a human can guess the Schrödinger's cat state in the closed system, assigning it a state either alive or dead, according to the subjective probability of his creed, and because the quantum reality to him is what the wavefunction assigns to be in reality.

Such reasoning affirms that a quantum state is just a subjective probability assignment, and the reality of the quantum world tosses the observer's beliefs to elect that one most evident.

This is hard to accept for the traditional scientific thought, "It is really hard to believe something you don't actually believe" [8], yet for a QBist it is a consistency question, considering the subjective probability a logic extension of the truth table.

That extended logic encourages us to generalize the quantum reasoning to the macroscopic world, as we find it explicitly in decision system methodologies.

We concern the collapsed wavefunction after observation (or measurement) which is characterized by subjective probabilities, and so the observers' personal information gives us expectations or degrees of belief.

When I look at the world nature my mind interprets detected cues by sensing and perception of the natural world as appearances in correspondence to reality.

Whether I don't get information signals from the reality, this reality exists but I can't say anything about it, and whether I detect some real signals I will build a creed on reality, assembling captured cues in my mind and weighting them to obtain the most evident subjective probability to make decisions.

This is the apparent reality or the reality we think it is.

Consequently, the matter on macroscopic world relies on the subjective probability, as well as it must occur on the quantum level when it is observed (or measured).

That's a question of believing, but to believe grounding on a probability induced by the own reality.

The behaviour of the observed quantum world is the same as that of our macroscopic world, taking in account the reality reveals the same physical principles.

All this means that the natural interpretation of the reality is the reality itself, even when it exists inside a closed quantum system or whenever we are not observing it, and so the material substance is nothing more than a human judgement, reasoning on detected signals radiated from matter to process the subjective probability according to the intrinsic character of things.

The reality remains "the thing-in-itself", as Immanuel Kant (1724-1804) said, however to me it is the appearance I judge to be the reality in extremely rapid decision processes.

We arrive to the conclusion that the reality exists as it is, but our consciousness decides its appearance using a very pragmatic heuristic, estimating some cues by perception in very short time, and seeking the accumulated evidence to adopt cue weights for diagnosticy and owing to their credibility proportions for each competitive hypothesis, integrating both weights in products as evidence factors, and choosing the evident hypothesis with the maximum sum of those factors ${ }^{10}$.

${ }^{10}$ The decision theory defines a heuristic based on the cue diagnostic $[p(E)]$, that ranks detected cues with normalized weights according to their importance for the decision making, and also on the cue credibility $[p(E \mid H)]$ of each hypothesis representing cue confidences by their impact weights, as we can see in the following table with three cues, and choosing the best hypothesis between two competitors (hypothesis A and B), defining evidence factors $[p(E) \cdot p(E \mid H)]$ for each cue and each competitor by the product of diagnosticity weights $\left(W_{\mathrm{di}}\right)$ and credibility weights $\left(W_{\mathrm{ci}}\right)$, and electing the maximum sum of evidence factors:

\begin{tabular}{|c|c|c|c|c|c|}
\hline \multirow{2}{*}{$\begin{array}{c}\text { Diagnose } \\
\text { cue }\end{array}$} & \multirow{2}{*}{$\begin{array}{c}\text { Diagnosticy } \\
\text { weight }\end{array}$} & \multicolumn{2}{|c|}{ Credibility weight } & \multicolumn{2}{c|}{ Evidence factor } \\
\cline { 3 - 6 } & Hypothesis A & Hypothesis B & Hypothesis A & Hypothesis B \\
\hline cue 1 & $W_{\mathrm{d} 1}$ & $W_{\mathrm{Ac} 1}$ & $W_{\mathrm{Bc} 1}$ & $W_{\mathrm{d} 1} \cdot W_{\mathrm{Ac} 1}$ & $W_{\mathrm{d} 1} \cdot W_{\mathrm{Bc} 1}$ \\
\hline cue 2 & $W_{\mathrm{d} 2}$ & $W_{\mathrm{Ac} 2}$ & $W_{\mathrm{Bc} 2}$ & $W_{\mathrm{d} 2} \cdot W_{\mathrm{Ac} 2}$ & $W_{\mathrm{d} 2} \cdot W_{\mathrm{Bc} 2}$ \\
\hline cue 3 & $W_{\mathrm{d} 3}$ & $W_{\mathrm{Ac} 3}$ & $W_{\mathrm{Bc} 3}$ & $W_{\mathrm{d} 3} \cdot W_{\mathrm{Ac} 3}$ & $W_{\mathrm{d} 3} \cdot W_{\mathrm{Bc} 3}$ \\
\hline \multicolumn{7}{|c}{ Sum of evidence factors } & Sum $_{\mathrm{A}}$ & Sum \\
\hline
\end{tabular}


This optimization heuristic translates the Bayesian probability into our everyday mental process, for instance buying a shirt or choosing a new car to buy it.

Whether the decision time is very short we feel acting under intuitive pulses, judging without any elaborate reasoning, and we can say the intuition feeling relies upon a very rapid decision making.

This is an interesting by-product of the present search.

The truth is that when we are observing our world we are always reasoning with subjective probability pulses.

I see the green forest because I detect light cues from the trees using my eyes (and the integrated biological vision system), and they convince me that the tree colour is green, matching the wave frequency ${ }^{11}$ of the light detected from trees with the learned language stored in my neural memory.

The consciousness answers to the subjective probability of the real wavefunction whenever the human observes the reality, because it appears as an open system, whose telonomy is the appearance of the reality that affects him.

Therefore, the observer is not separated from the observed [9], they both interact intimately.

It comes now the opportunity to ask if all systems are real or not.

Taking systems from the reality we observe them without any doubt, each concrete system belongs to the reality, doesn't matter it is a macroscopic or a quantum system, and its systemic model is also real, because I design it in a representative abstract nature but its functional operation corresponds to a concrete system.

A system model makes decisions exactly as the natural system itself, supposing systemic essentials are perfect in the case of simplex systems, or it approximates more or less right outputs in complex model systems with imperfect systemic essentials.

All this is what an observer can infer observing both natural and model realities and using detected cues from outside of both black boxes (of natural and model systems), because results are exactly the same for human consciousness if system outputs are equal, or we approach them as much as they seem to be similar.

For instance, a refrigerator's user doesn't need to know how the refrigerator system works, being only concerned on their cold outputs, but in the case of a refrigerator's designer he must be aware of its five systemic essentials to produce good equipments.

Concluding, a concrete (natural or model) system means a black box to the observer, and we don't need any especial knowledge about what is going on inside, supposing it operates as it was designed, because we reduce the reality to telonomy measurements and excluding the side-by-side information that doesn't matter to the intentionality target.

Beyond that, decision systems are also real?

As we know, in such situations we organize data sets which inform us on reality features, and we decide electing one of them through subjective probabilities, and the choice represents the most valuable or convincing hypothesis.

Only later real facts will confirm whether our choices are acceptable or unacceptable judgements.

In any case, the methodology is universal, based on wavefunction collapses by observation and using the Bayesian probability tool, i.e., a decision system is an abstract system but the systemic complexity governs their behaviour as well as in concrete natural systems.

This important corollary is proved by systemic essentials of decision systems analysing the character of detected cues.

The acrony is definite because in general we can fairly estimate all diagnosticity weights before comparing hypothesis; the axony is complete when we can clearly guess credibility weights of cues for competitive hypothesis; the aquadry is uncertain because it is possible missing some relevant cues, owing to the proper finiteness of cue boundaries; the adaptacy is determined in the case of impartial or not biased choices, otherwise it will come some errors by overconfidence bias and psychological anchoring heuristics, provoking undetermined optimum working points.

Following these main lines, normally a decision system is an open complex system, the telonomy being the best possible output from global weighted cues and their mutual relationships, although we cannot always assume the optimum decision.

What we must do is trying to reduce the degree of complexity, recollecting better information on systemic essentials, endeavouring to attain simplexities or at least approximating as much as possible to simplex systems.

Here it is the recommended strategy.

In conclusion, this approach lengthens the Copenhagen interpretation, which judges that reality is created by observation, defending the thesis it is much more than that by reasoning on the reality as a mind formulation founded on subjective probabilities.

${ }^{11}$ Here frequency means the number of wave cycles per second (not the frequency of occurrences in an arbitrary gap of time, which is used to define objective probabilities). 
Indeed, I can summarize the previous discussion in few basic items, raising important issues to be debated in general and in particular cases, as it follows:

1. The reality means my belief on decision making by perception of signals, which are radiated from things as system acrony (measurement problem).

2. I use a subjective probability scheme in my mind to decide what reality is, choosing the most convincing accumulated axony (subjective problem).

3. I can't know the total meaning of the observed reality, because the biological structure of my body limits every systemic aquadry (boundary problem).

4. The correlation among collected data and psychological bias and heuristics may deviate from the best choice, and I cannot assure attaining the optimum system adaptacy (optimum control problem).

5. Common decisions in our current life are intuitive, we make them in extremely short time and so the reality seems apparently to be evident from the system telonomy (consciousness problem).

We are now seeing why I am convinced that Kant's appearance of reality is complex under the systemic standpoint, and so I hope we will be able to handle complex systems of worldwide realities developing the systemic complexity theory by scientific methodologies.

Actually, science research must get down to essential fundamentals, and science theory consists of human rational attempts to represent both appearance and reality of things and ideas.

Are reality and appearance a unique thing?

The science and technology will develop detecting tools for supra-human sensing contacts with non-observable reality by humans, and we research on human-machine interfaces [10] to integrate simplexicity into complexity of nature systems inserting trends to observe the reality itself in a new systemic supra-appearance.

That is my conviction, which emerges from decision making in my mind based on some detected signals and memory ones.

In reality, that's the reality.

\section{REFERENCES}

[1] A. North Whitehead, Adventures of Ideas, Cambridge at the University Press, London, 1933.

[2] Nick Herbert, Quantum Reality. Beyond the New Physics, Archor Books, New York, 1985.

[3] John Ziman, Real Science, What it is, and what it means, Cambridge University Press, Cambridge, 2000.

[4] Hermínio Duarteramos, "Hard and soft systems intentionality", $7^{\text {th }}$ Congress of UES, 2006, Lisbon; also in Res-Systemica, n. ${ }^{\circ}$, AFSCET.

[5] G. T. Arecchi, "Pattern generation and competition in non-equilibrium optical media: a case study for complexity", in The Physics of Complex Systems, IOS Press, Oxford, 1997.

[6] David Lindley, Where does the Weirdness go?, Basic Books, New York, 1996.

[7] Max Born, Albert Einstein, The Born-Einstein Letters, Walter and Company, New York, 1971.

[8] Christopher Fuchs, Interview with a Quantum Bayesian, arXiv:1207.2141v1[quant-ph] 9 Jul 2012; also in Maximilian Schlosshauer, Elegance and Enigma: The Quantum Interviews, Springer, Frontiers Collection, 2011.

[9] David Bohm, On Dialogue (1996), Routledge, London, 2014.

[10] R. Antunes, F. V. Coito, H. Duarteramos, "Skill evaluation in point-to-point human-machine operation", Applied Mechanics and Materials, Vol. 394, 2013, Trans Tech Publications, Zurich-Durten.

\section{KEYNOTE SPEAKER}

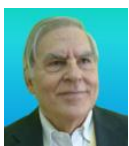

Hermínio Duarteramos was born in July 20, 1936. He got the PhD degree researching dielectric physics at Leibniz University in Hannover (Germany), and he did work during thirty years as full professor in the New University of Lisbon (Portugal), being President of the Scientific Council (1993-1996). Leading the Systemic Engineering Group, he did teach graduate and post-graduate students on control theory and practice, based on neural networks and fuzzy logic, especially on man-machine integrated systems, and coordinated some research projects on cognitive control and decision systems. He was co-founder of three universities (Luanda University in 1972, New University of Lisbon in 1976, New Athena Senior University in 2008), and was Committee's President for Portuguese terminology of the International Electrotechnical Commission (2004-2007), and President of the Portuguese Association for Systemic Complexity APOCOSIS (2006-2009). Being emeritus professor since 2006, nowadays he is researching on systemic complexity of worldwide integrated systems. 
Acta Europeana Systemica $\mathrm{n}^{\circ} 4$ 